\title{
Immunohistochemical Diagnosis of Hirschsprung's Disease and Allied Disorders
}

\author{
Valerija Zuikova*, Ivanda Franckevica*,**, Ilze Strumfa*, Ivars Melderis** \\ *Riga Stradins University, Riga, Latvia \\ ${ }^{* *}$ Children's Clinical University Hospital, Riga, Latvia
}

\begin{abstract}
SUMMARY
Introduction. Hirschsprung's disease accounts for approximately $20 \%$ of neonatal bowel obstruction and has a mortality rate of 20 to $25 \%$. As the pathology severely affects a child's quality of life, quick and precise diagnosis is mandatory. For years, diagnosis relied completely on histopathological analysis of rectal biopsies using haematoxylin-eosin and acetylcholinesterase stains. However, there have been many attempts to find an immunohistochemical marker that would simplify diagnosis of Hirschsprung's disease. Acceptable markers should be highly sensitive and specific, easy to use and reliable.
\end{abstract}

Aim of the study. The aim is to disclose the full morphological scope of Hirschsprung's disease and allied disorders by using data available at the Children's Clinical University Hospital (Riga, Latvia) and to identify the most sensitive and specific immunohistochemical marker for the diagnosis of Hirschsprung's disease and allied disorders.

Material and methods. In a retrospective study, all patients diagnosed with Hirschsprung's disease and allied disorders at the Pathology Bureau of the Children's Clinical University Hospital between April 2010 and October 2014 were identified. Immunohistochemical visualisation of calretinin, chromogranin A and synaptophysin was carried out. A conjugated polymer system EnVision was used to detect the bound primary antibodies. The study was controlled by findings in the bowel wall of adults and children lacking aganglionosis. The intensity and pattern of ganglion cell and nerve fibre staining were evaluated semi-quantitatively on a scale of one to three in both submucosal and myenteric plexuses of bowel. The specificity and sensitivity of each immunohistochemical marker were determined with a MedCalc online calculator.

Results. During the relevant period, Hirschsprung's disease (23) and allied disorders (12) were diagnosed in 35 patients. Among these children, $45.7 \%$ were diagnosed during the first year of life. Three types of Hirschsprung's disease were present - short segment disease $(73.9 \%)$, long segment disease $(21.8 \%)$ and total colonic aganglionosis $(4.3 \%)$. Allied Hirschsprung's disorders were of three different types - hypoganglionosis $(75 \%)$, zonal aganglionosis $(16.7 \%)$ and immaturity of ganglion cells $(8.3 \%)$. After evaluation of the reactivity of immunohistochemical markers, calretinin, synaptophysin and chromogranin showed staining intensity of ganglion cells of $2.47,0.58$ and 0.63 , respectively, while nerve plexus staining intensity was 1.84 for calretinin, 2.68 for synaptophysin and 1.79 for chromogranin. Calretinin was characterised by sensitivity/ specificity as high as $90.5 \% / 92.9 \%$ while these parameters were only $52.4 \% / 100.0 \%$ for chromogranin $A$ and $33.3 \% / 14.3 \%$ for synaptophysin.

Conclusions. In Latvia, the diagnosis of Hirschsprung's disease or allied disorder is made later in life than it is in other countries. Hirschsprung's disease was more frequent than the allied disorders. It can present as short segment disease, long segment disease and total colonic aganglionosis. Calretinin showed the highest reactivity to ganglion cells and proved to be the most specific and sensitive marker for diagnosis of Hirschsprung's disease and allied disorders. Synaptophysin showed strong staining of myenteric plexuses and can be used for assessment of extrinsic nerve fibres despite its low reactivity to ganglion cells.

Key words: Hirschsprung's disease, immunohistochemistry, calretinin

\section{INTRODUCTION}

Hirschsprung's disease is a developmental disorder characterised by the absence of parasympathetic intramural ganglion cells of submucosal and myenteric plexuses in the rectum and varying lengths of bowel proximal to the rectum (20). It occurs in approximately one in every 5000 live births, accounts for approximately $20 \%$ of neonatal bowel obstructions and has a mortality rate of 20 to $25 \%(7,23)$. The pathological background of Hirschsprung's disease is defective craniocaudal migration of vagal neural crest cells during embryogenesis or their degeneration after migration that results in aganglionosis or hypoganglionosis of the bowel (5). The term 'allied Hirschsprung's disorder' covers a number of conditions in which patients experience all or some clinical signs of Hirschsprung's disease, but histologically there are some ganglion cells present (5). In hypoganglionosis, the symptomatic patient has a reduced number of ganglion cells above the dentate line (20). Zonal agangliosis describes a bowel with narrow zones of aganglionosis that are surrounded by normal ganglion cells below and above aganglionic segments (5). This condition is rarely seen and is believed to be caused by ischaemia, viral disease or immunologic injury (20). Immaturity of ganglion cells is characterised by normal or increased ganglion cell count, but ganglion cells and their nuclei are slightly smaller and without a recognisable nucleolus. Although immaturity of ganglion cells has mostly been seen in neonates and premature infants and often improves with maturity, it may, however, persist (18).

The diagnosis of Hirschsprung's disease requires proof of the absence of submucosal and myenteric ganglion 
cells from examined bowel segments (11). There are several histological techniques for evaluating rectal biopsy for the presence of ganglion cells. Haematoxylineosin stain (H\&E) is one of the most available and commonly used methods. It requires a large number of serial sections of each biopsy in order to establish the diagnosis of Hirschsprung's disease $(10,11,22)$. However, diagnosis with H\&E staining is not always possible, because it has limitations in term of examining those submucosal areas where ganglion cells are small and irregularly distributed. This technique is also limited as regards diagnosis of immature ganglion cells in neonates. H\&E interpretation requires a skilled and experienced pathologist and is very time-consuming because of the necessity to interpret many sections before a biopsy can be confirmed as negative $(10,22)$. Acetylcholinesterase (AChE) histochemistry is the second most widely used method. Histochemical demonstration of AChE positive cholinergic nerve fibres within the lamina propria provides supportive evidence for the diagnosis of Hirschsprung's disease. The presence of thick fibres between the crypts in lamina propria is regarded as highly specific for diagnosis (23). The biggest problem with AChE staining is the fact that changes may not be clearly evident in a patient younger than six months, especially in shortsegment Hirschsprung's disease. Additionally, this technique requires frozen sections and the staining procedure must be followed precisely with the use of fresh-prepared reagents (7).

Immunohistochemical stains have the advantage of using sections from paraffin embedded biopsies, thus eliminating the need to obtain extra biopsies (7). Many immunohistochemical markers have been tested for diagnosis of Hirschsprung's disease. Immunohistochemical stains that have been used to highlight the changes in Hirschsprung's disease are calretinin, neuron specific enolase, synaptophysin, CD56, cathepsin D, vimentin, bcl2, S100 and chromogranin A (16). Most of these have been proved to be diagnostically insignificant, but some are shown to have diagnostic value.

Calretinin is a vitamin D-dependent calcium-binding protein that is involved in calcium signalling, thus playing an important role in the organisation and functioning of the central nervous system $(6,13)$. The precise functions of calretinin are still unclear (2). Recent studies have demonstrated the importance of calretinin stain in diagnosis of Hirschsprung's disease and allied disorders (12). Calretinin displays positive nerve staining in the submucosa, muscular layer of mucosa and often even in lamina propria. In addition, calretinin shows strong staining of ganglion cells and slight positive staining of Schwann cells. In the case of Hirschsprung's disease there is a total absence of staining in all layers of bowel. Some cases of Hirschsprung's disease can present slightly positive staining in large nerve fibres without ganglion cell staining (6).
Synaptophysin is a synaptic vesicle glycoprotein that is present in presynaptic vesicles of neurons and in neuroendocrine cells (26). Synaptophysin antibody shows reactivity to presynaptic vesicles of neurons in brain, spinal cord, neuromuscular junctions, retina and vesicles of adrenal gland and pancreatic islets (15). Some authors report that synaptophysin can play an important role in diagnosis of Hirschsprung's disease and allied disorders thanks to its ability to demonstrate enteric innervation of the bowel (4). On the other hand, other studies have found no reliable proof that synaptophysin can provide better distinction in differential diagnosis of Hirschsprung's disease, although it can support AChE investigation (19).

Chromogranin $\mathrm{A}$ is a secretory protein that can be found in vesicles of neuroendocrine, endocrine, neuronal cells and sympathetic nerves (14, 21). Chromogranin A stain is primarily used in the identification of neuroepithelial and neuroendocrine differentiation in normal and neoplastic tissues as well as neuronal elements in brain and bowel (14). It has been reported that in Hirschsprung's disease chromogranin A immunoreactive adrenergic fibres were present in muscle layers of aganglionic segment. The concentration of chromogranin A in smooth muscle specimens was higher in an aganglionic bowel compared with a normal ganglionic bowel (24).

\section{AIM OF THE STUDY}

The aim of the study was to disclose the full morphological scope of Hirschsprung's disease and allied disorders by using data available at the Children's Clinical University Hospital (Riga, Latvia) and to detect which of the three immunohistochemical markers (calretinin, synaptophysin, chromogranin) is the most sensitive and specific in the diagnosis of Hirschsprung's disease and allied disorders.

\section{MATERIAL AND METHODS}

A retrospective study of consecutive patients diagnosed for Hirschsprung's disease and allied disorders at the Pathology Bureau of the Children's Clinical University Hospital between April 2010 and October 2014 was carried out. It yielded 35 paediatric patients with diagnosis of Hirschsprung's disease and allied disorders. From these, a representative group was selected for immunohistochemical visualisation (IHC) in order to compare the sensitivity and specificity of the selected markers. The patients were selected for IHC depending on the availability and amount of histological material. Overall, IHC was carried out on 16 histological samples from 14 paediatric patients. In addition, intestinal wall specimens from 10 adults and 9 children from the Pathology Institute of Pauls Stradins Clinical University Hospital (Riga, Latvia) and the Pathology Bureau of the Children's Clinical University Hospital were used as two control groups. In all control subjects, the diagnosis of Hirschsprung's disease and allied disorders was excluded after unequivocal identification of 
ganglion cells. In total, 34 paraffin-embedded tissue blocks were examined and analysed using H\&E and three potentially important immunohistochemical markers. There is no ethical conflict in this report; the principles outlined in the Declaration of Helsinki were followed by the authors.

All tissue samples were fixed in 10\% neutral buffered formalin and embedded in paraplast blocks (Diapath S.r.l., Bergamo, Italy). For both H\&E and IHC examination, $3 \mu \mathrm{m}$ thick sections were obtained. For IHC, primary antibodies for detection of calretinin (clone DAK-Calret 1, monoclonal mouse anti-human antibody, Dako, Glostrup, Denmark), chromogranin A (clone DAK-A3, monoclonal mouse anti-human antibody, Dako) and synaptophysin (clone SY38, monoclonal mouse anti-human antibody, Dako) were used. A conjugated polymer system EnVision (Dako) was used to detect the bound primary antibodies. Immunohistochemical staining was performed manually.

The intensity and pattern of ganglion cells and nerve fibre staining were evaluated semi-quantitatively by light microscopy both in submucosal and in myenteric bowel layers. IHC reactivity in ganglion cells was evaluated as follows: 0 - no staining or impossible to visualise ganglion cell, 1 - mild staining, 2 moderate staining, 3 - strong staining. In ganglion cells, the staining was evaluated both in nucleus and in cytoplasm. Reactivity for staining of neural plexuses was evaluated by an analogous scale.

The obtained data were analysed using IBM SPSS Statistics 20.0 (IBM, USA). Descriptive (mean, standard deviation (SD) and median) and analytical statistics (statistical significance) were implemented. Differences were considered significant if $p<0.05$. To measure specificity and sensitivity, a MedCalc online calculator (MedCalc Software, Belgium) was applied.

\section{RESULTS}

During the investigated period, 35 cases with Hirschsprung's disease and allied disorders were identified. Patient age varied from three days to 15 years (mean \pm SD, $49 \pm 55$ months; median 17 months). Among 35 patients, 16 were diagnosed during the first year of their life $(45.7 \%)$, while 19 were diagnosed later $(54.3 \%)$. In three patients $(8.6 \%)$, Hirschsprung's disease or allied disorders was disclosed during the newborn period. There were 23 cases of Hirschsprung's disease $(65.7 \%)$ while 12 patients were diagnosed with allied disorders $(34.3 \%)$. Three types of Hirschsprung's disease were present: 17 patients were diagnosed with short segment disease $(73.9 \%)$, five with long segment disease $(21.8 \%)$ and one with total colonic aganglionosis $(4.3 \%)$. Allied Hirschsprung's disorders included hypoganglionosis in nine patients $(75 \%)$, zonal aganglionosis in two patients (16.7\%) and immaturity of ganglion cells in one patient $(8.3 \%)$. From 35 patients with morphologically confirmed
Hirschsprung's disease or allied disorder, a representative group was selected for IHC to compare the sensitivity and specificity of three different immunohistochemical markers. This study group comprised 16 samples taken from 14 paediatric patients. Patient age varied from three days to 12 years (mean $\pm \mathrm{SD}, 30 \pm 46$ months). The tissues were represented by samples obtained by aspiration biopsy, laparoscopic biopsy and biopsy during laparotomy. In cases where patients underwent both rectal suction biopsy and intraoperative seromuscular biopsy, the latter was chosen. From 16 samples used in IHC analysis of sensitivity and specificity of the selected markers, seven were obtained during rectal suction biopsy $(44 \%)$, three during laparoscopic biopsy (19\%) and six were biopsies during laparotomic surgery $(37 \%)$.

In the immunohistochemically studied paediatric patient group, 12 patients presented with Hirschsprung's disorder $(85.7 \%)$ and two others were diagnosed with hypoganglionosis $(14.3 \%)$. Regarding the types of Hirschsprung's disease, there were nine cases of short segment disease $(75 \%)$, two cases of long segment disease $(16.7 \%)$ and one case of total colonic aganglionosis $(8.3 \%)$, as shown in Figure 1 .

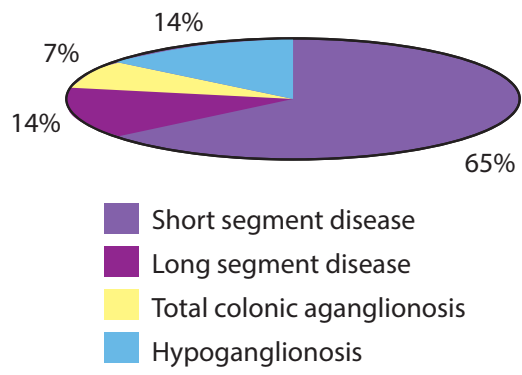

Fig. 1. Types of Hirschsprung's disease and allied disorders in immunohistochemically evaluated paediatric patient group

The paediatric control group consisted of nine samples taken from nine paediatric patients without bowel innervation defects undergoing treatment in the Children's Clinical University Hospital. Male to female ratio was $6: 3$ or 2.0. Patient age varied from four days to 10 years (mean \pm SD, $24 \pm 43$ months) These samples were obtained by aspiration biopsy (two cases, 22\%), laparoscopic biopsy (five cases, $56 \%$ ) and biopsy during laparotomy (two cases, $22 \%)$. Biopsies were obtained from sigmoid colon $(67 \%)$, rectum $(22 \%)$ and ileum $(11 \%)$. Patients clinical diagnoses were: chronic constipation in four patients $(45 \%)$, necrotising enterocolitis in one patient (11\%), intestinal malrotation in one patient $(11 \%)$, umbilical polyp in one patient $(11 \%)$, anal atresia in 
one patient (11\%) and abdominal adhesions in one patient $(11 \%)$. In all obtained biopsies ganglion cells were unequivocally found in both submucosal and myenteric layers of bowel.

There was no significant statistical difference in the age distribution between paediatric patient and control groups $(p=0.69)$. The difference in gender distribution between the two groups was also statistically insignificant $(\mathrm{p}=0.29)$.

The adult control group consisted of 10 samples from 10 patients lacking bowel innervation defects. In all obtained biopsies ganglion cells were unequivocally found in both submucosal and myenteric layers of bowel. Male to female ratio was 6:4 or 1.5. Patient age varied from 51 years to 83 years (mean \pm SD, $67 \pm 10$ years). All the biopsies were obtained from the sigmoid colon during laparoscopic or laparotomic surgery. Histological findings resulted in seven patients being diagnosed with sigmoid tumour $(70 \%)$, one with perforation of the sigmoid colon $(10 \%)$ and two with anastomotic ulcer after resection of the sigmoid colon $(20 \%)$.

In order to compare three selected immunohistochemical markers, the degree of ganglion cell staining versus extrinsic nerve fibre staining (Table 1) in the paediatric control group and the adult control group was detected. Ganglion cell presence in control subjects was confirmed via microscopy of H\&E stained samples (Figures 2 and 3).

Table 1. Reactivity of the investigated immunohistochemical markers

\begin{tabular}{|l|l|l|l|}
\hline $\begin{array}{l}\text { Immuno- } \\
\text { histochemical } \\
\text { reactivity }\end{array}$ & Calretinin & Synaptophysin & Chromogranin A \\
\hline Ganglion cells & 2.47 & 0.58 & 0.63 \\
\hline $\begin{array}{l}\text { Nerve plexus } \\
\text { staining }\end{array}$ & 1.84 & 2.68 & 1.79 \\
\hline
\end{tabular}

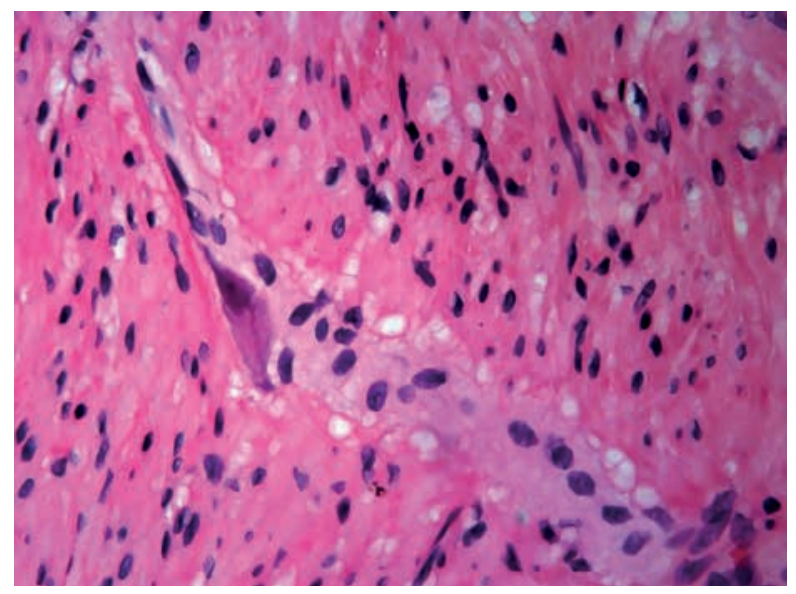

Fig. 2. Enteric nerve plexus showing ganglion cell in H\&E stain. Magnification (x400)

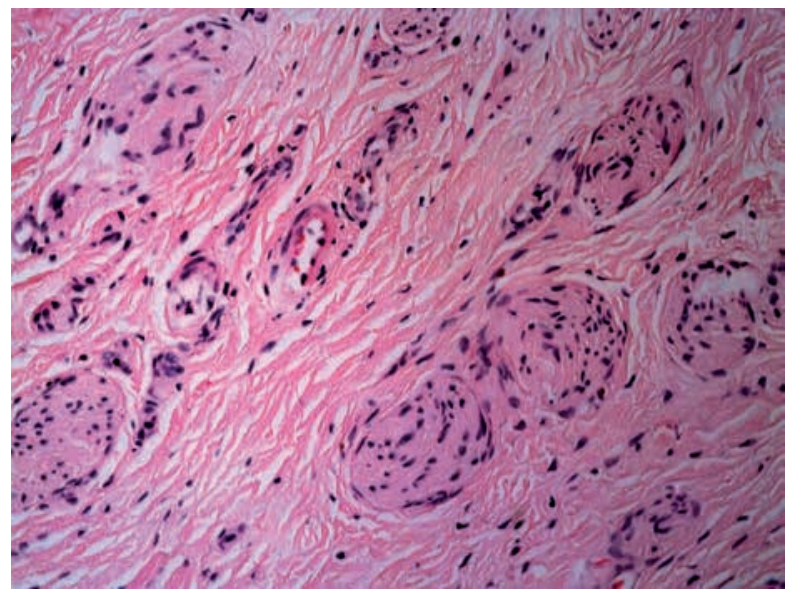

Fig. 3. Enteric nerve plexuses lacking ganglion cells in H\&E stain. Magnification (x200)

IHC (Table 1) showed that calretinin yielded sufficient cytoplasmic and nuclear staining of ganglion cells, while maintaining acceptably low nerve plexus staining (Figures 4 and 5).

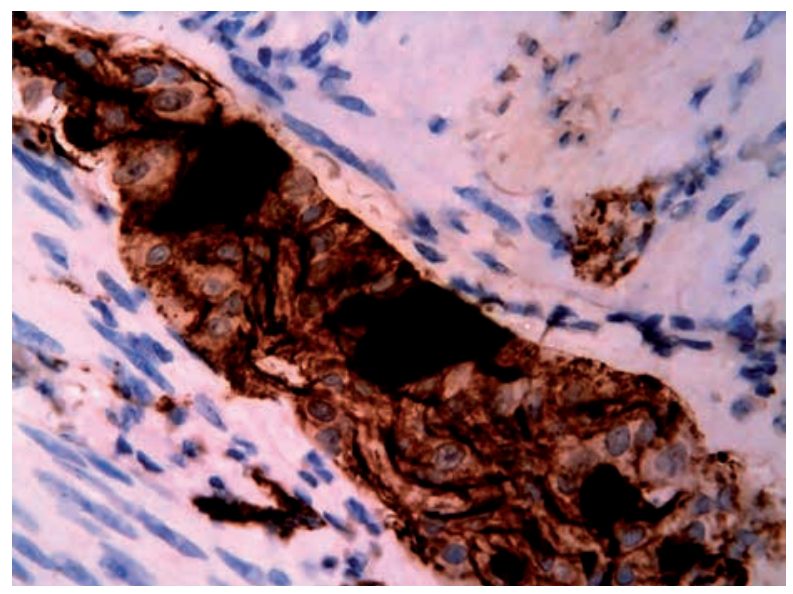

Fig. 4. Enteric nerve plexus with ganglion cells by calretinin stain. Magnification (x400)

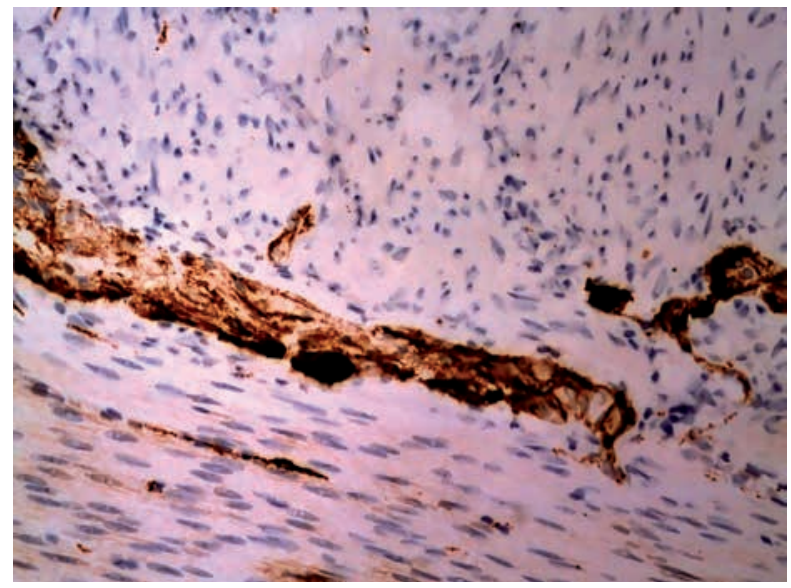

Fig. 5. Enteric nerve plexus showing ganglion cells by calretinin stain. Magnification (x200) 
Synaptophysin showed a high degree of extrinsic nerve fibre staining. Strong nerve plexus staining concealed the submucosal and myenteric ganglion cells. Strong staining of myenteric plexuses makes synaptophysin a good diagnostic marker for hypertrophic or hypotrophic changes of extrinsic nerve fibres (Figures 6 and 7).

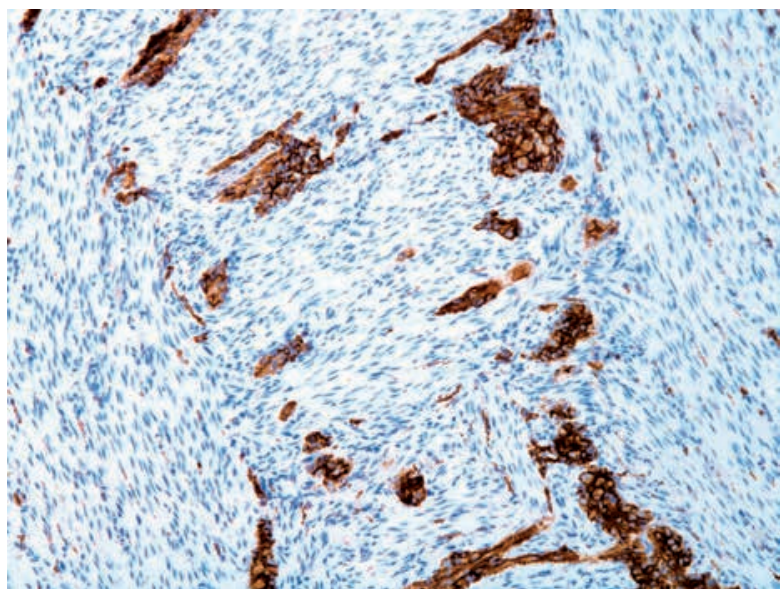

Fig. 6. Normal structure of enteric nerve plexus in a control person by synaptophysin stain. (x100)

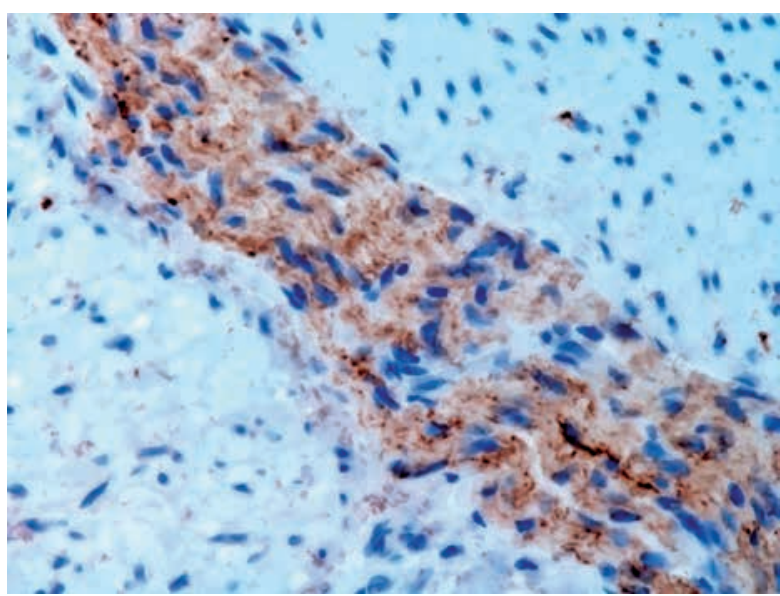

Fig. 7. Enteric nerve plexus of patient with Hirschsprung's disease by synaptophysin stain. (x400)

Chromogranin A showed low nuclear and cytoplasmic ganglion cell staining while maintaining acceptable reaction with extrinsic nerve fibres and diffusely staining all neural and neuroendocrine structures of the bowel wall (Figures 8 and 9).

Thus, calretinin is the only IHC marker with sufficiently high reactivity to ganglion cells in both submucosal and muscle layers of bowel. Synaptophysin can be a useful marker for assessment of extrinsic nerve fibres despite its low reactivity to ganglion cells. Chromogranin A showed low reactivity to ganglion cells, making it useless in detection and diagnosis of Hirschsprung's disease and allied disorders.

Additionally, the sensitivity and specificity of ganglion

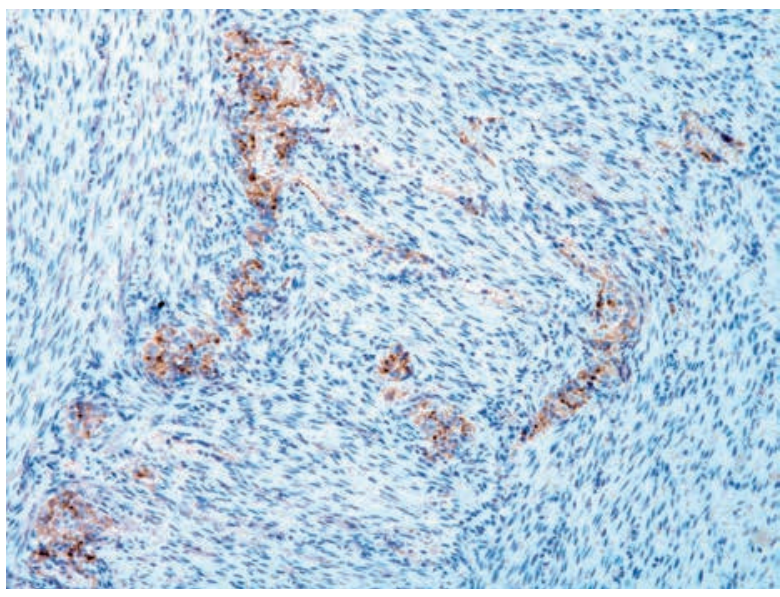

Fig. 8. Normal structure of enteric nerve plexus in a control person by chromogranin A stain. (x100)

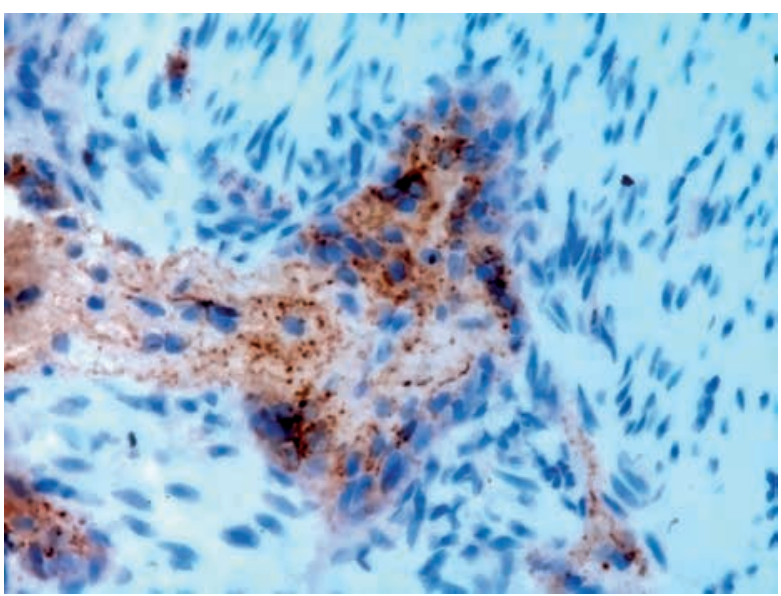

Fig. 9. Enteric nerve plexus of patient with Hirschsprung's disease by chromogranin A stain. (x400)

cell detection for each of the immunohistochemical markers were evaluated (Table 2). True positive cases were defined as samples from the control group with positive IHC staining. True negative cases were defined as all samples from the patient group without positive IHC staining. False positives were all patient biopsies exhibiting positive IHC staining. False negatives were all control samples lacking positive IHC staining. 
Table 2. Sensitivity and specificity of the investigated immunohistochemical markers

\begin{tabular}{|l|l|l|l|}
\hline Parameter & Calretinin & Synaptophysin & Chromogranin A \\
\hline Sensitivity & $90.5 \%$ & $33.3 \%$ & $52.4 \%$ \\
\hline Specificity & $92.9 \%$ & $14.3 \%$ & $100.0 \%$ \\
\hline
\end{tabular}

Of all three evaluated markers calretinin showed the highest sensitivity and specificity, reaching positive predictive value of $90.5 \%$ and negative predictive value of $92.9 \%$. Together with high ganglion reactivity, this confirms calretinin as the most valuable immunohistochemical marker of the three evaluated. Chromogranin A showed $100 \%$ specificity, but only $52.4 \%$ sensitivity, meaning that in patients with aganglionosis it showed no signs of ganglion cells, but many false negative results in control patients were observed. Low sensitivity together with low reactivity to ganglion cells makes chromogranin A useless in diagnosis of Hirschsprung's disease and allied disorders. Synaptophysin showed both low sensitivity and specificity, making it the least useful of the three immunohistochemical markers for evaluating the presence of ganglion cells in the bowel. However, it can be used for evaluation of hypertrophy or hypotrophy of extrinsic nerve fibres.

\section{DISCUSSION}

Hirschsprung's disease and allied disorders are common pathologies in neonates, infants and even older paediatric patients. Hirschsprung's disease occurs in 1:5000 live births and is three to four times more common in males than females $(1,23)$. Age of diagnosis of Hirschsprung's disease and allied disorders varies depending on the source. Data from a nationwide survey in Japan show that in $48.7 \%$ of patients diagnosis was made during the first month of life (9). A prospective survey by the Australian Paediatric Surveillance Unit reported that diagnosis of Hirschsprung's disease was made during the newborn period in $90.5 \%$ of patients (25). Our data showed that $45.7 \%$ of patients were diagnosed during the first year of their life while $54.3 \%$ were diagnosed after the age of one year. Only in $8.6 \%$ of all cases was diagnosis made in the newborn period. Thus, data obtained during the present study showed later diagnosis of Hirschsprung's disease compared with Japan and Australia. This could be because the present study included cases of allied Hirschsprung's disorders that can develop symptoms and consequently be diagnosed later than total colonic aganglionosis (3).

Among the 35 studied patients, there were 23 cases of Hirschsprung's disease $(65.7 \%)$ while 12 patients were diagnosed with allied disorders $(34.3 \%)$. A study conducted in Pakistan showed similar distribution between Hirschsprung's disease and allied disorders (8). There were three types of Hirschsprung's disease present: $73.9 \%$ of patients were diagnosed with short segment disease, $21.8 \%$ were diagnosed with long segment disease and $4.3 \%$ of all cases had total colonic aganglionosis. This spectrum is similar to the literature data (7).

The present study concentrated on finding a suitable immunohistochemical marker for diagnosis of Hirschsprung's disease and allied disorders. Three markers were selected (calretinin, synaptophysin and chromogranin A) on the basis of their ability to react with different neural structures in the bowel wall. In order to detect the most suitable stain for diagnosis of Hirschsprung's disease, markers were compared on their reactivity to ganglion cells and background neural structures, their sensitivity and specificity.

Among the tested diagnostic markers, calretinin showed the best reactivity to ganglion cells (2.47), sensitivity $(90.5 \%)$ and specificity $(92.9 \%)$. Compared with AChE, which is still considered the gold standard in diagnosis of Hirschsprung's disease, calretinin is more sensitive $(90.5 \%$ versus $85 \%)$ throughout all age groups and is more suitable for diagnosis of allied Hirschsprung's disorders. While its specificity is slightly lower than that reported regarding AChE (92.9\% versus 100\%) it still has an important role in diagnosis of Hirschsprung's disease and allied disorders thanks to its clear and simple stain pattern and ability to use formalin-fixed, paraplast-embedded samples $(6,17)$. The other two immunohistochemical markers (synaptophysin and chromogranin A) showed lower sensitivity, specificity and reactivity to ganglion cells, making them less reliable and useful diagnostic markers for Hirschsprung's disease and allied disorders, although synaptophysin showed strong staining of myenteric plexuses which makes it a good diagnostic marker for hypertrophic or hypotrophic changes of extrinsic nerve fibres.

In conclusion, our study shows that calretinin is a highly sensitive and specific marker and may be a better choice than other immunohistochemical markers, or H\&E and AChE for diagnosis of Hirschsprung's disease and allied disorders.

\section{CONCLUSIONS}

1. In Latvia, diagnosis of Hirschsprung's disease or allied disorder is made later in life than in other countries, with $45.7 \%$ of patients being diagnosed during the first year of their life and $54.3 \%$ after the age of one year.

2. Hirschsprung's disease occurred more often than allied Hirschsprung's disorders and was found in three different histological patterns: short segment disease, long segment disease and total colonic aganglionosis.

3. According to the results of the study, calretinin showed the highest reactivity to ganglion cells and proved to be the most specific and sensitive marker for diagnosis of Hirschsprung's disease and allied disorders.

4. Synaptophysin showed strong staining of myenteric plexuses and can be used for assessment of extrinsic nerve fibres despite its low reactivity to ganglion cells. 
5. Chromogranin A showed low reactivity to ganglion cells, making it useless in detection and diagnosis of Hirschsprung's disease and allied disorders.

\section{ACKNOWLEDGEMENT}

The present study was carried out within the frames of scientific project ZP08 (Riga Stradins University)

\section{Conflict of interest: None}

\section{REFERENCES}

1. Badner JA, Sieber WK, Garver KL, Chakravarti A. A genetic study of Hirschsprung disease // Am J Hum Genet, 1990; 46:568 - 580

2. Billing-Marczak K, Kuznicki J. Calretinin- sensor or buffer- function still unclear // Pol J Pharmacol, 1999; 51:173- 178

3. Collins $\mathrm{MH}$, Boid JT, Sheridan R. Gastrointestinal System // In: Epstein JI, Husain AN. Biopsy Interpretation of Paediatric Lesions. 1st ed. Philadelphia: Wolters Kluwer Health; 2014; 1 - 37

4. Corsois L, Boman F, Sfeir R, Besson R, Gottrand F, Boccon-Gibod L. Synaptophysin expression abnormalities in Hirschsprung's disease // Ann Pathology, 2004; 24:407 - 415

5. Day DW, Jass JR, Price AB, Sheperd NA, Sloan JM, Talbot NJ, Warren BF, Williams GT. Normal embryology and fetal development; developmental abnormalities of the large intestine and the anal region // In: Morson and Dawson's Gastrointestinal Pathology. 4th ed. Oxford: Wiley-Blackwell; 2003; $445-451$

6. Guinard-Samuel V, Bonnard A, De Lagausie P, Philippe-Chomette P, Alberti C, El Ghoneimi A, Peuchmaur M, Berrebi-Binczak D. Calretinin immunohistochemistry: a simple and efficient tool to diagnose Hirschsprung disease // Mod Pathol, 2009; 22:1379 - 1384

7. Hart J, Wilcox R, Weber CR. The gastrointestinal tract // In: Stocker TJ, Dehner LP, Husain AN. Stocker \& Dehner's Paediatric Pathology. 3rd ed. Philadelphia: Lippincott Williams \& Wilkins; 2010; $574-640$

8. Henna N, Nagi AH, Sheikh MA, Shaukat M. Morphological patterns in children with ganglion related enteric neuronal abnormalities // J Ayub Med Coll Abbottabad, 2011; 23:14 - 17

9. Ikeda K, Goto S. Diagnosis and treatment of Hirschsprung's disease in Japan. An analysis of 1628 patients // Ann Surg, 1984; 199:400 - 405

10. Kapur RP. Immunohistochemistry and the diagnosis of Hirschsprung's disease // Am J Clin Pathol, 2006; $126: 9-12$

11. Kapur RP. Intestinal motor disorders // In: Russo P, Ruchelli E, Picolli DA. Pathology of Paediatric Gastrointestinal and Liver Disease. 2nd ed. New York: Springer; 2014; $249-316$

12. Kapur RP, Reed RC, Finn LS, Patterson K, Johanson J, Rutledge JC. Calretinin immunohistochemistry versus acetylcholinesterase histochemistry in the evaluation of suction rectal biopsies for Hirschsprung disease // Pediatr Dev Pathol, 2009; 12:6 - 15

13. Leong ASY, Cooper K, Leong FJWM. Calretinin // In: Leong ASY, Cooper K, Leong FJWM. Manual of Diagnostic Antibodies for Immunohistology. 2nd ed. London: Greenwich Medical Media; 2003; 45 48

14. Leong ASY, Cooper K, Leong FJWM. Chromogranin // In: Leong ASY, Cooper K, Leong FJWM. Manual of Diagnostic Antibodies for Immunohistology. 2nd ed. London: Greenwich Medical Media; 2003; 159 160

15. Leong ASY, Cooper K, Leong FJWM. Synaptophysin // In: Leong ASY, Cooper K, Leong FJWM. Manual of Diagnostic Antibodies for Immunohistology. 2nd ed. London: Greenwich Medical Media; 2003; 405 406

16. Mackenzie JM, Dixon MF. An immunohistochemical study of the enteric neural plexus in Hirschsprung's disease // Histopathology, 1987; 11:1055 - 1066

17. Martucciello G, Pini Prato A, Puri P, Holschneider AM, Meier-Ruge W, Jasonni V, Tovard JA, Grosfeld JL. Controversies concerning diagnostic guidelines for anomalies of the enteric nervous system: A report from the fourth international symposium on Hirschsprung's disease and related neurocristopathies // J Pediatr Surg, 2005; 40: $1527-1531$

18. Moore SW. Neurocristopathies and particular associations with Hirschprung's disease // In: Holschneider AM, Puri P. Hirschsprung's Disease and Allied Disorders. 3rd ed. New York: Springer; 2008; $253-256$

19. Patt S, Stoltenburg-Didinger G. Immunohistochemical studies using synaptophysin in intestinal biopsies in Hirschsprung disease // Zentralbl Pathol, 1992; 138:325 - 329

20. Petras RE, Gramlich TE. Non-neoplastic intestinal diseases // In: Mills SE, Carter D, Greenson JK, Reuter VE, Stoler MH. Sternberg's Diagnostic Surgical Pathology. 5th ed. Philadelphia: Lippincott Williams \& Wilkins; 2009; 1313 - 1368

21. Prestifilipo A, Blanco G, Vitale MP, Giuffrida D. Chromogranin A and neuroendocrine tumors // In: Lowell A. Neuroendocrine Tumor. Croatia: InTech; 2012; $11-17$

22. Rosai J. Hirschprung's disease and related disorders // In: Rosai J. Rosai and Ackerman's Surgical Pathology. 9th ed. St Louis, MO: Mosby; 2004; $777-779$

23. Sebire NJ, Malone $M$, Ashworth M, Jacques TS. Gastrointestinal neuromuscular/motility diseases // In: Sebire NJ, Malone M, Ashworth M, Jacques TS. Diagnostic Paediatric Surgical Pathology. 1st ed. London: Churchill Livingstone; 2009; 561 - 578

24. Shen Z, Larsson LT, Malmfors G, Oberg K, Eriksson B, Sundler F. Chromogranin A and B in neuronal elements in Hirschsprung's disease: An immunocytochemical and radioimmunoassay study // J Pediatr Surg, 1994; 29:1293- 1301 
25. Singh SJ, Croaker GD, Manglick P, Wong CL, Athanasakos H, Elliott E, Cass D. Hirschsprung's disease: the Australian Paediatric Surveillance Unit's experience // Pediatr Surg Int, 2003; 19: $247-250$

26. Weidenmann B, Franke W, Kuhn C, Moll R, Gould V. Synaptophysin: A marker protein for neuroendocrine cells and neoplasms // Proc Natl Acad Sci U S A, 1986; 83: 3500 - 3504

\section{Address:}

Ivanda Franckevica,

Children's Clinical University Hospital,

Vienibas gatve 45, Riga, LV-1004, Latvia

e-mail: ivanda.franckevica@gmail.com 